\title{
PREPRINTS NA COMUNICAÇÃO CIENTÍFICA: UMA INTRODUÇÃO
}

\author{
EDILSON DAMASIO*
}

\begin{abstract}
RESUMO
O modelo de publicação em preprints está inserido na comunicação científica. Definido como um documento técnico-científico disponibilizado de forma preliminar antes de sua publicação. Após os recursos da Internet e com diretrizes indicadas pelos movimentos de Acesso Aberto, surgiram, na década de 1990, os primeiros projetos de disponibilização e arquivamento de artigos científicos antes da publicação, denominados preprints. O primeiro servidor, o arXiv é muito utilizado por áreas de exatas e computação há duas décadas. No início de 2010, os preprints começaram a ganhar vida em outras áreas e, em 2013, na área biomédica, com a criação do bioRXiv, com o objetivo de compartilhar textos, rascunhos, documentos de trabalho e seus resultados antes da revisão por pares e publicação. O modelo de publicação preprints está em constante crescimento junto às publicações em acesso aberto, e mostra-se como um novo paradigma da revisão por pares e uma das atuais discussões em comunicação científica e editoração, chegando a ser um modelo adotado pelas principais revistas interdisciplinares do mundo, as revistas Nature e Science, e com projeto lançado em 2017 para o SciELO. Também são incentivados por agências de financiamento e autores da área biomédica como um modelo muito positivo, prioritário e eficiente.Outra facilidade dos preprints é a sua possibilidade de conexão com outros modelos de disponibilização de conteúdos atuais, como as redes sociais e outras mídias, gerando uma visualização e impacto nos meios de divulgação não tradicionais.
\end{abstract}

PALAVRAS-CHAVE: Preprints. Comunicação científica. Editoração. Publicação científica.

\begin{abstract}
The preprints publishing is a model on scientific communication with as a pre-disseminations scientific and technical documents. The Internet resources and the Open Access-OA movement emerged in the 1990s the first project to release de articles before to the publication. The first server, arXiv is a model to more 20 years an used for scientific areas on exact sciences and computing, and in 2013 started to biomedical fields with the creation of bioRXiv, the
\end{abstract}

*Doutor em Ciência da Informação pela Universidade Federal do Rio de Janeiro/IBICT. Bibliotecário do Departamento de Matemática da UEM. 
purpose of sharing texts, drafts, working papers, reports and their results prior to peer review and publication. The preprints model is constantly growing in OA model, and show as a new paradigm of peer review, and the current global discussions in scientific communication and publishing, get to a model adopted by most interdisciplinary journals Nature and Science and started on 2017 on SciELO project. Are encouraged by funders and the biomedical authors as a very positive, priority and efficient model. Another feature of preprints its ability to connect with other availability of current model, to content such as social shares and other media, generating a view and impact in non-traditional publishing models.

KEYWORDS: Preprints. Scientific communication. Publishing.

\section{INTRODUÇÃO}

Atualmente, em nível nacional e internacional, existem grandes discussões sobre a comunicação científica disponibilizada em preprints. Esse modelo de comunicação já está moldado em Acesso Aberto devido à mudança e às novas reformulações e alterações conforme as demandas da Tecnologias de Informação e Comunicação (TICs) às necessidades dos leitores. A Ciência tem sua própria vida, por si, já se altera universalmente e, assim, também as metodologias para a sua comunicação. Os atores principais, nesse universo, são a academia e os pesquisadores. A pesquisa e seus resultados, formulações de novas técnicas e metodologias, os resultados encontrados, originais ou não, estudos comparativos, novas teorias, novas discussões sobre teorias já definidas anteriormente, novos pensadores, novas interfaces no meio científico necessitam de sua comunicação e seguem novos modelos e métodos de publicação como os artigos em plataformas hipermídia, com gráficos e vídeos interativos, anotações vinculadas ao conteúdo, comentários, formatos XML, dentre outras funcionalidades que esse modelo suporta.

Apresentaremos uma breve revisão bibliográfica em comunicação científica e informação, com objetivos, meios, e discussões atuais. Para esse fim, serão apresentadas algumas bases conceituais da discussão do Acesso Aberto nas décadas de 1990 e 2000, seus principais objetivos alcançados, seguido de aspectos discutidos na área de Ciência da Informação. Finalizará com uma breve apresentação dos artigos disponibilizados em formato preprints, formato de disponibilização da produção com avaliação por pares aberta e compartilhada. 


\section{COMUNICAÇÃO CIENTÍFICA NOVOS FORMATOS}

Arthur J. Meadows (1999), autor em estudos sobre comunicação científica, aborda que a comunicação junto a novas tecnologias é uma preocupação constante no meio acadêmico, devido à exigência de comunicar aos pares os resultados das pesquisas. Preocupação essa de cientistas, bibliotecários e editores, e pesquisadores.

Destaca o autor que as discussões acerca da comunicação no mundo globalizado têm pontos primordiais, a apresentação e divulgação das recentes descobertas nas sociedades científicas, das diferentes Ciências, em suas diversas matérias e particularidades.

[...] a Comunicação Científica como um processo que engloba a produção da informação, sua inserção nos canais de comunicação utilizados pelas comunidades científicas - que podem ser formais (como a informação por escrito e que apresenta uma necessidade de validação, através do sistema peer review) ou informais (que são canais de comunicação orais ou as fontes de informação primárias, que não chegaram a ser validadas) - e a recuperação dessa informação pelos pesquisadores e acadêmicos (MEADOWS, 1999; MUELLER, 1994 apud BIOJONE, 2003, p. 21-22).

Segundo Meadows (1999), a Comunicação Científica tem os seguintes objetivos principais:

- comunicar aos membros e pares no meio científico novos estudos, mostrando que estão disponíveis para serem aferidos, utilizados por outros, e, principalmente, avaliados;

- disseminar, no meio acadêmico/científico, os resultados parciais ou finais de estudos empíricos, métodos ou técnicas aplicadas;

- partilhar, de maneira universal, pesquisas recém finalizadas à comunidade científica e seu público, que são os pesquisadores e cientistas, e estes agindo como os avaliadores.

As históricas tradições de publicação da pesquisa foram alteradas, devido às mudanças das publicações formais em papel para o novo mundo eletrônico que, nas décadas de 1970 e 1980, advindas desse meio, tornaram-se, com a utilização de Tecnologias da Informação e Comunicação (TICs), mais desenvolvidas, culminando com a Internet e os acessos on-line desde a década de 1990 e agora a interoperabilidade de metadados. 
As Tecnologias da Informação e Comunicação (TICs) e suas diferenças nas matérias (campos científicos) a serem apresentadas e disseminadas aos pesquisadores cresceram nas últimas décadas. Neste momento, tem destaque para o emprego das TICs, métodos e modelos que se transformam e moldam-se em áreas específicas de ciências biomédicas, exatas na Física, Matemática e Computação. Culminando, atualmente, com um modelo de publicação preprints, como uma das possibilidades de antecipação do processo de revisão por pares e revisão por pares aberta e compartilhada.

\section{DA PUBLICAÇÃO ELETRÔNICA E OS PREPRINTS}

Os pesquisadores têm definido, em seus objetivos, o que pesquisar, e como deve utilizar e apresentar os resultados encontrados. Sua disseminação e, principalmente, sua discussão no meio acadêmico, o objeto e objetivos, o motivo e os tipos de pesquisas. As cobranças de produtividade e qualidade são medidas, há décadas, pelos modelos/metodologias métricas de análise da produção científica. Esses são alguns dos fatores principais que influenciam as pesquisas e indicam onde devem ser publicados seus resultados.

Os fluxos de divulgação da Informação nos meios científicos são importantes desde o formato em papel até os canais eletrônicos atuais, e, atualmente, as necessidades de canais eletrônicos para a busca da informação e comunicação decorrem, principalmente, da utilização de redes eletrônicas e os leitores, ou seja, a informação organizada já em canais atualizados pelas TIC's e em Acesso Aberto.

A apresentação no evento aparentemente serviu como uma excelente oportunidade para integrar os resultados de um com a frente de pesquisa atual. Dois outros meios de comunicação (colegas não-locais e preprints) (GARVEY; LIN; TOMITA, 1972, p. 272, tradução nossa) $)^{1}$.

O processo e o fluxo editorial é parte da comunicação e do fazer do pesquisador, que necessita gerenciá-lo, desde como

\footnotetext{
${ }^{1}$ Meeting presentations were useful sources of information more often during the final stage, especially when it came to relating and integrating findings to ongoing or recently completed work. The meeting presentation apparently served as an excellent opportunity to integrate one's findings with the current research front. Two other media (non-local colleagues and preprints).
} 
escrever, prazos, sua importância, a produção científica e para quem publicar, até o peer review (revisão por pares) e publicação final.

Está em crescimento exponencial o modelo de publicação em Acesso Aberto antes da revisão por pares ser finalizada, os preprints que, historicamente, foram utilizados por alguns campos científicos em ciências exatas e tem por objetivo da circulação do artigo ou paper para colegas e pesquisadores antes da publicação, é uma pré-impressão de manuscrito, rascunho e/ou documento de trabalho. Devido a essa característica, pode ser atualizado e reenviado a qualquer momento pelos autores (PREPRINTS.ORG, 2018). Apesar de ser não usual, em muitos campos científicos, na área de ciências exatas, é comum e nessa década iniciando nas biomédicas, humanas, e outras áreas multidisciplares e estão em constante crescimento.

FIGURA 1 - Fluxo editorial de revisão por pares/Fluxo do preprints. ${ }^{2}$
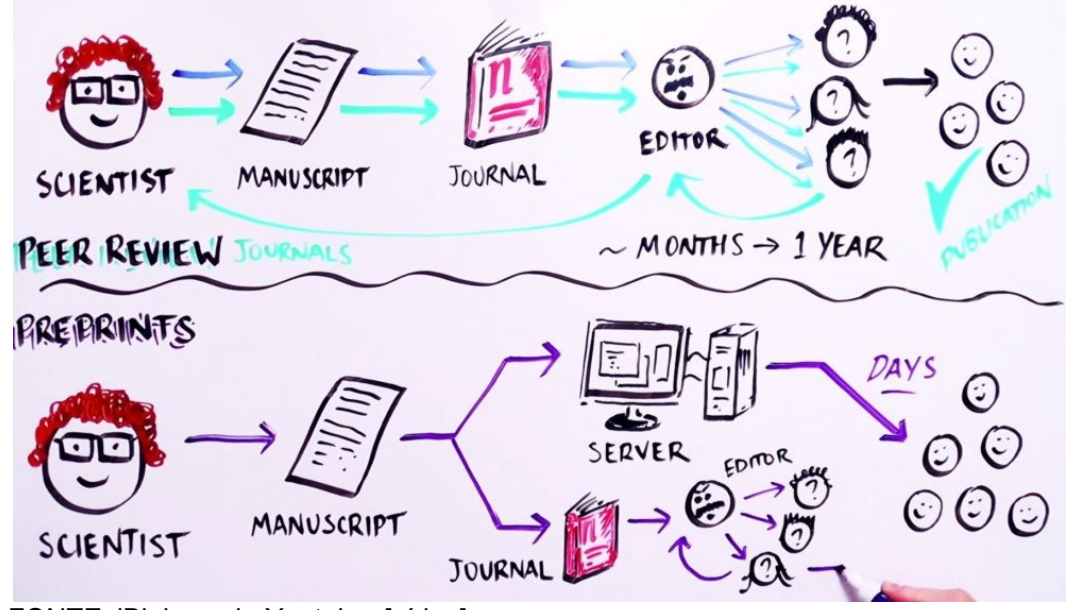

FONTE: iBiology via Youtube. [vídeo]

Podemos identificar, na figura 1, a apresentação de duas vias/modelos de acesso à revisão por pares; na primeira parte (superior), o fluxo editorial, através de revisão por pares tradicional, que pode se estender a meses ou anos até a publicação. Na parte inferior da figura, é visualizado o exemplo do fluxo editorial com

${ }^{2}$ Vídeo do processo de preprints, realizado pela iBiology - What are preprints? Disponível em: <https://youtu.be/2zMgY8Dx9co>. Acesso em: 20 maio 2017. 
preprints, em que os autores enviam para o servidor de preprints e, posteriormente, ou ao mesmo tempo, o conteúdo para avaliação pela revista no modelo tradicional, que, em poucos dias, já estará disponível à comunidade científica. Nesse momento, a revisão por pares acontece de duas formas distintas e com a possibilidade até de antecipação do processo.

Historicamente, os preprints seriam um meio de comunicação em eventos que mudou para o formato eletrônico com a utilização de servidores eletrônicos, para 'armazenamento', e da Internet, como 'acesso'.

Segundo Paul Ginsparg (2011), mesmo considerados uma infraestrutura nova de publicação, os preprints vêm se desenvolvendo de maneira lenta, acompanhando um processo semelhante à passagem da impressão em papel para o formato digital. Um dos motivos é por ser uma metodologia que está absorvendo a mudança do formato PDF para o formato XML, que suporta várias funcionalidades, como: gráficos manipuláveis, visualização dinâmica, anotações vinculadas aos artigos, comentários e marcação semântica. Para isso, o autor mostra que deve existir o mesmo cuidado em manter um arquivo estável na literatura, conforme os publicados em papel durante séculos.

Dentre os métodos de modelos, podemos definir que são servidores de arquivamento e distribuição de pesquisas: seus dados, artigos, imagens, relatórios. Existe controle de submissão e existe moderação dos comentários aos preprints. Como modelo de Acesso Aberto, podem suportar a antecipação de parcela da revisão por pares aberta e compartilhada.

Ewen Callaway (2017), em artigo publicado na revista Nature, mostra que o crescimento do número de preprints estão em crescimento contínuo, e aumentou 3 vezes dos anos de 2013 a 2016. Importante destacar que o autor apresenta outros servidores de preprints, vinculados a projetos, agências de fomento, como: PeerJPreprints; F1000 Reserch; Nature Precedings; The Winnower; Preprints.org; Wellcome Open Research.

Em pesquisa realizada no ano de 2016 com 392 pesquisadores pelo projeto ASAPbio (Accelerating Science and Publication in biology), junto a grupo focal da comunidade em Biologia e biomédicas da utilização e o papel que os preprints poderiam desempenhar na comunicação científica. Os resultados mostraram que mais de $90 \%$ dos respondentes estão muito satisfeitos com a disponibilização em preprints. Como uma experiência muito positiva, e que, no futuro, estarão enviando 
novamente seus textos em preprints. Segundo o survey, é um modelo incentivado pelas agências de fomento e prioritário para a divulgação de suas pesquisas, e consequentemente revistas e agências promovem essa representação da produtividade das pesquisas (ASAPbio, 2016).

\subsection{Preprints: alguns modelos}

O maior banco de dados de preprints do mundo é resultado de projeto iniciado na década de 1990, em Los Alamos National Laboratory, no Novo México, EUA, com o nome de arXiv, que, após vinte anos de existência, é um modelo estável e bem sucedido com 700 mil textos completos acumulados e com um número de crescimento de 9.448/mês e 113.380 no ano de 2016. Agora hospedado na Cornell University Library de Nova York e subsidiado por fundações. Nesses arquivos, permanece a versão arquivada no projeto e também o link para o acesso aos papers já publicados em revistas científicas (arXiv, 2016; GINSPARG, 2011).

Na figura 2, é mostrado o aumento acumulado do número de submissões às áreas de cobertura do arXiv em áreas de exatas e computação, pode-se identificar que existe um crescimento exponencial nesse modelo de publicação que duplicou em menos de oito anos, de 2008 a 2016. 
FIGURA 2 - Submissões cumulativas no ArXiv, de 1991-2016.

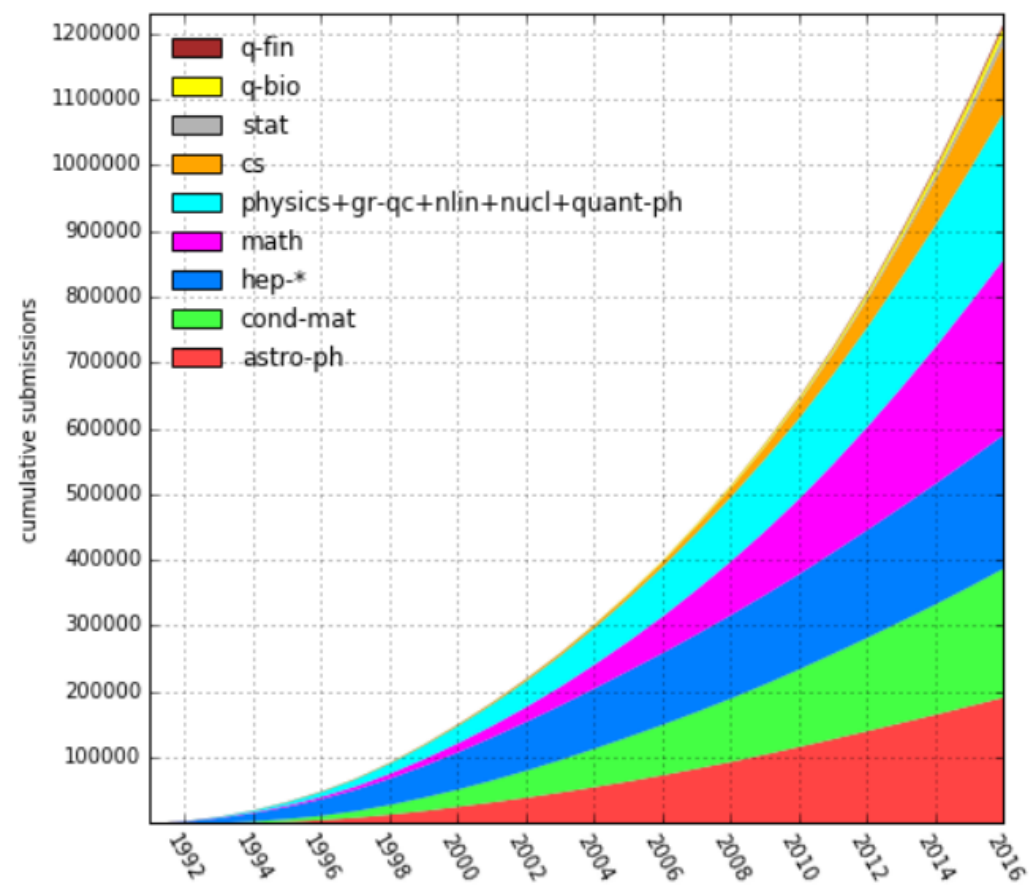

FONTE: ArXivstatistics (2016).

Pode-se destacar na Figura 2 que a área de Matemática (math) com o maior número de submissões, 267.469 (21,9\%), seguido de HEP (High Energy Physics=Física de Alta Energia) $203.315 \quad(16,7 \%)$, Cond-Mat (Condensed Matter=Matéria Condensada) $196.411(16,1 \%)$ e Astro-ph (Astro Physics=Astro Física) 192.160 (15,8\%).

Desde o ano de 2013, os preprints começaram a ganhar vida na área biomédica, com a criação do servidor bioRXiv, lançado pela Cold Spring Harbor Laboratory Press, em Nova York, que opera de maneira semelhante ao ArXiv para o compartilhamento de papers em semanas ou meses antes da publicação formal.

É importante mostrar que muitas revistas biomédicas publicadas por editores tradicionais e por sociedades alteraram suas políticas para permitir a prática da utilização de preprints, como exemplo: as revistas da Genetics Society of America, Ecologycal Society of America, Nature Publishing Group, Cell Press e a revista 
Science (CALLAWAY, 2013).

Na figura 3, é apresentado um registro de artigo de revisão disponibilizado no bioRXiv, que ainda não foi finalizada a revisão por pares e publicado em um período inferior a 30 dias desde a sua primeira versão. Essa metodologia comporta vários resultados métricos relacionados ao artigo. Esse preprint publicado em 8 de dezembro de 2018, constava em 6663 acessos ao resumo, 1718 acessos ao PDF e a disponibilização de informação de acessos na modalidade de altimetria com 470 (altmetrics) e 751 menções no Twitter. Vejamos que existe uma grande quantidade de acessos ao artigo devido a fatores como a importância do tema peer review, o tema e/ou área do assunto em Comunicação científica e Educação (KLAUS; DEL ALAMO, 2018).

FIGURA 3 - Artigo no bioRXiv com dados métricos/altimétricos/redes sociais

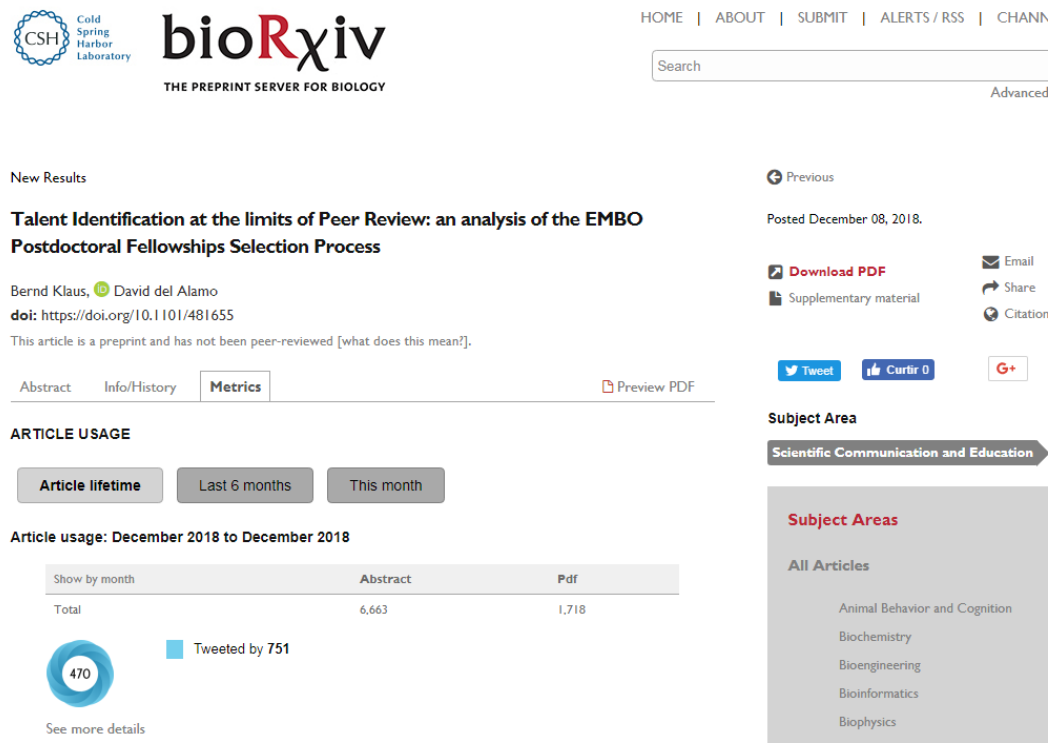

FONTE: doi: https://doi.org/10.1101/481655 Klaus e Del Alamo (2018).

Em informação atual, mostramos que o modelo de preprints já foi adotado para a utilização na plataforma de links persistentes do Crossref, em maio de 2016, como uma metodologia de atribuição realizada pelos membros associados ao Crossref, em que o DOI (Digital Object Identifier) será diferente do DOI utilizado pelo editor 
na publicação final. São documentos diferentes, mas que têm uma relação em que os metadados fazem a 'união' através de uma resolução múltipla (CROSSREF, 2018).

Existem servidores de preprints multidisciplinares, sendo o servidor Preprints.org.com sede na cidade de Basel, Suíça e vinculado a editora MDPI de revistas em Acesso Aberto. Na figura 4, mostram-se as áreas de abrangência desse servidor em Ciência \& Tecnologia, Engenharias, Humanas, Sociais aplicadas, dentre outras.

FIGURA 4 - Preprints.org e suas áreas de cobertura

\begin{tabular}{|c|c|c|c|c|c|}
\hline $\begin{array}{l}\text { ARTS \& HUMANITIES } \\
\text { Anthropology \& } \\
\text { Ethnography } \\
\text { Archaeology } \\
\text { Art History \& Restoration } \\
\text { Comparative Literature } \\
\text { General Humanities } \\
\text { Hilstory } \\
\text { Uinguistics } \\
\text { Uterary Studies } \\
\text { Media Studies } \\
\text { Music Studies } \\
\text { Philosophy } \\
\text { Theory of Art } \\
\text { Reiligious Studies } \\
\text { Other } \\
\text { Architecture and Design }\end{array}$ & $\begin{array}{l}\text { BEHAVIORAL } \\
\text { SCIENCES } \\
\text { Applied Psychology } \\
\text { Behavioral Neuroscience } \\
\text { Cognitive \& Experimental } \\
\text { Psychology } \\
\text { Cinical Psychology } \\
\text { Developmental Psychology } \\
\text { General Psychology } \\
\text { Social Psychology } \\
\text { Other }\end{array}$ & $\begin{array}{l}\text { BIOLOGY } \\
\text { Anatomy \& Morphology } \\
\text { Agricultural Sclences \&. } \\
\text { Agronomy } \\
\text { Animal Sciences \& Zoology } \\
\text { Ecology } \\
\text { Entomology } \\
\text { Forestry } \\
\text { Horticulture } \\
\text { Physiology } \\
\text { Plant Sciences } \\
\text { Other }\end{array}$ & $\begin{array}{l}\text { CHEMISTRY } \\
\text { Analytical Chemistry } \\
\text { Applied Chemistry } \\
\text { Chemical Engineering } \\
\text { Electrochemistry } \\
\text { General \&. Theoretical } \\
\text { Chemistry } \\
\text { Organic Chemistry } \\
\text { Inorganic \& Nuclear } \\
\text { Chemistry } \\
\text { Medicinal Chemistry } \\
\text { Physical Chemistry } \\
\text { Food Chemistry } \\
\text { Other }\end{array}$ & $\begin{array}{l}\text { EARTH SCIENCES } \\
\text { Atmospheric Science } \\
\text { Geochemistry \& Petrology } \\
\text { Geoinformatics } \\
\text { Geology } \\
\text { Geophysics } \\
\text { Oceanography } \\
\text { Palaeontology } \\
\text { Space Science } \\
\text { Environmental Sciences } \\
\text { Other }\end{array}$ & $\begin{array}{l}\text { ENGINEERING } \\
\text { Automotive Engineering } \\
\text { Blomedical \&. Chemical } \\
\text { Engineering } \\
\text { Civil Engineering } \\
\text { Control \& Systems } \\
\text { Engineering } \\
\text { Electrical \& Electronic } \\
\text { Engineering } \\
\text { Energy \& Fuel Technology } \\
\text { General Engineering } \\
\text { Industrial \&. Manufacturing } \\
\text { Engineering } \\
\text { Marine Engineering } \\
\text { Mechanical Engineering } \\
\text { Other } \\
\text { Construction }\end{array}$ \\
\hline $\begin{array}{l}\text { LIFE SCIENCES } \\
\text { Biochemistry } \\
\text { Biophysics } \\
\text { Biotechnology } \\
\text { Cell \& Developmental } \\
\text { Biology } \\
\text { Endocrinology \& } \\
\text { Metabolomics } \\
\text { Genetics } \\
\text { Immunology } \\
\text { Molecular Biology } \\
\text { Microbiologg } \\
\text { Virology } \\
\text { Other }\end{array}$ & $\begin{array}{l}\text { MATERIALS SCIENCE } \\
\text { Biomaterials } \\
\text { General Materials Science } \\
\text { Metallurgy } \\
\text { Nanotechnology } \\
\text { Polymers \& Plastics } \\
\text { Surfaces, Coatings \& Films } \\
\text { Other }\end{array}$ & $\begin{array}{l}\text { MATHEMATICS \& } \\
\text { COMPUTER SCIENCE } \\
\text { Algebra \& Number Theory } \\
\text { Analysis } \\
\text { Applied Mathematics } \\
\text { Artfifial Intelligence \&. } \\
\text { Robotics } \\
\text { Computational Mathematics } \\
\text { General Mathematics } \\
\text { Geometry \& Topology } \\
\text { Information Technology \& } \\
\text { Data Management } \\
\text { Logic } \\
\text { Numerical Analysis \& } \\
\text { Optimization } \\
\text { General \& Theoretical } \\
\text { Computer Science } \\
\text { Other } \\
\text { Probability and Statistics }\end{array}$ & $\begin{array}{l}\text { MEDICINE \& } \\
\text { PHARMACOLOGY } \\
\text { Allergology } \\
\text { Anesthesiology } \\
\text { Behavioral Neuroscience } \\
\text { Cardiology } \\
\text { Clinical Neurology } \\
\text { Dermatology } \\
\text { Nursing \& Health Studies } \\
\text { Gastroenterology } \\
\text { General Medical Research } \\
\text { Nutrition } \\
\text { Ophthalmology } \\
\text { Oncology \& Oncogenics } \\
\text { Pediatrics } \\
\text { Pharmacology \& Toxicology } \\
\text { Psychiatry \& Mental Health } \\
\text { studies } \\
\text { Sport Sclences \& Therapy } \\
\text { Urology } \\
\text { Veterinary Medicine } \\
\text { Other }\end{array}$ & $\begin{array}{l}\text { PHYSICAL SCIENCES } \\
\text { Acoustics } \\
\text { Applied Physics } \\
\text { Astronomy \& Astrophysics } \\
\text { Atomic \& Molecular Physics } \\
\text { Condensed Matter Physics } \\
\text { Fuids \& Plasmas } \\
\text { General \& Theoretical } \\
\text { Physics } \\
\text { Mathematical Physics } \\
\text { Nuclear \& High Energy } \\
\text { Physics } \\
\text { Optics } \\
\text { Particle \&. Field Physics } \\
\text { Radiation \& Radiography } \\
\text { Other }\end{array}$ & $\begin{array}{l}\text { SOCIAL SCIENCES } \\
\text { Accounting } \\
\text { Economics } \\
\text { Econometrics \& Statistics } \\
\text { Education Studies } \\
\text { Finance } \\
\text { Geography } \\
\text { Law } \\
\text { Ubrary \& Information } \\
\text { Sclence } \\
\text { Marketing } \\
\text { Microeconomics and } \\
\text { Decision Sciences } \\
\text { Organizational Economics \& } \\
\text { Management } \\
\text { Political Science } \\
\text { Sociology } \\
\text { Other } \\
\text { Business and Administrative } \\
\text { Sciences }\end{array}$ \\
\hline
\end{tabular}

FONTE: Prepints.org (2018).

$\mathrm{Na}$ figura 5, mostra-se uma comunicação publicada no Preprints.org de artigo da área de Biblioteconomia e Ciência da Informação. Pode-se identificar que ele está em uma primeira versão e é uma comunicação em formato de resumo, e com um ano de publicação no formato de preprints. O preprint teve 2073 visualizações e foi baixado 576 vezes seu texto completo. Esse modelo do Preprints.org mostra não somente o resumo, ou um gráfico, mas também outras possibilidades de identificação de acesso ao artigo, como ser revisado por pares através de outras 
plataformas, como o Publons, plataforma de revisão por pares da Web Of Science, com autorização do editor ou revisores, possibilitam ser disponibilizados os pareceres pré ou pós-publicação (PUBLONS, 2018).

FIGURA 5 - Comunicação publicada no Preprints.org de Library \&Information Science

preprints.org > social sciences > library\& information science > doi: 10.20944/preprints201706.0111.v1
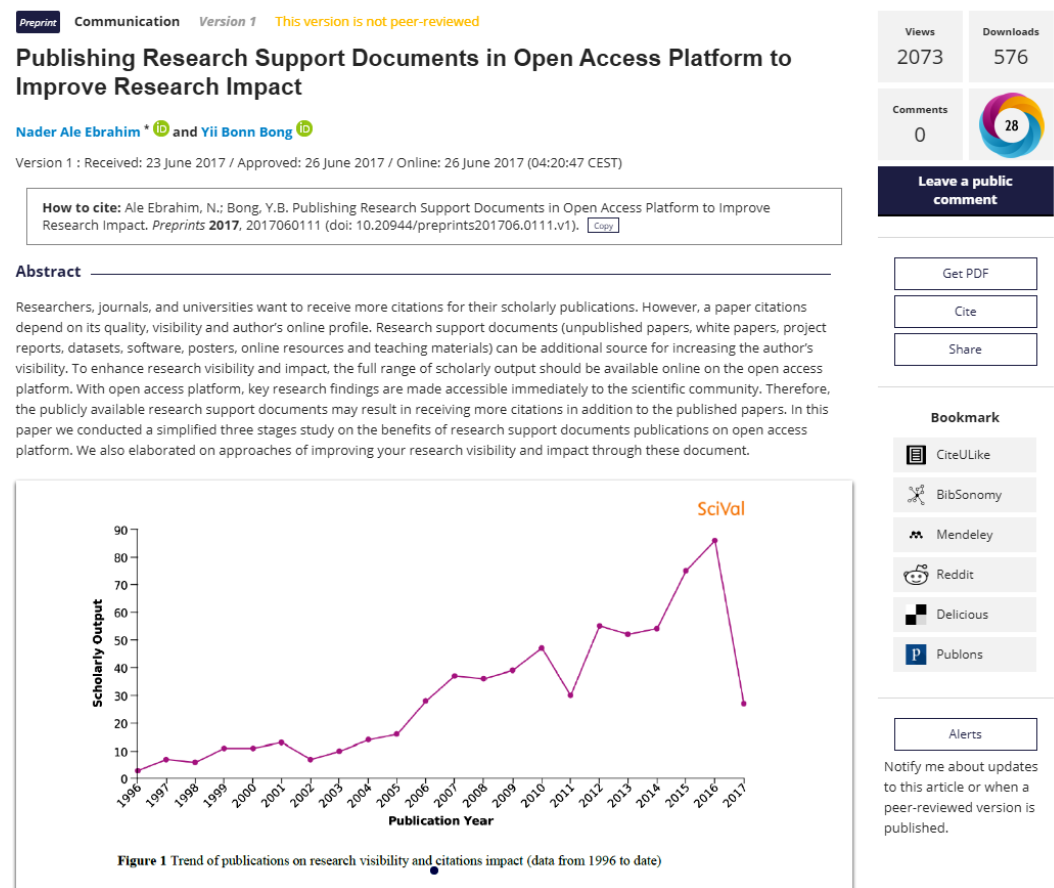

Abstract

Researchers, journals, and universities want to receive more citations for their scholarly publications. However, a paper citations

depend on its quality, visibility and author's online profile. Research support documents (unpublished papers, white papers, project reports, datasets, software, posters, online resources and teaching materials) can be additional source for increasing the author's platform. With open access platform, key research findings are made accessible immediately to the scientific community. Therefore the publicly avaliable research support documents may result in receiving more citations in addition to the published papers. In this paper we conducted a simplified three stages study on the benefits of research support documents publications on open access
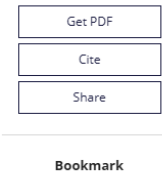

圆 CiteULike

$x^{3}$ Bibsonomy

n. Mendeley

(5) Reddit

- Deliciou

P

Publons

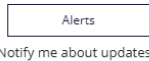

to this article or when a

peer-reviewed version is published.

FONTE:doi: https://doi.org/10.20944/preprints201706.0111.v1 Prepints.org (2018).

Geoffrey Bilder (2016) afirma que é um formato de apoio aos modelos de publicação atuais, que estão em mutação, voltados para mostrar os registros de citações acadêmicas para um documento intelectual único, e não voltado para versões diferentes.

No Brasil, em fevereiro de 2017, o projeto SciELO (Scientific Electronic Library Online) apresentou um planejamento para a utilização de preprints em suas publicações, com a utilização de servidor próprio, denominado SciELO Preprints. Packer, Santos e Meneguini (2017), afirmam que "O objetivo principal é contribuir para acelerar a disponibilização dos resultados de pesquisa e posicionar 
a comunicação científica dos países que participam da Rede SciELO, e em particular seus periódicos, em sintonia com os avanços e importância crescente da publicação de preprints internacionalmente" (PACKER; SANTOS; MENEGHINI, 2017).

Em editoração, identificou-se que a pré-publicação pode deixar uma possibilidade de antecipação de citações ao artigo, como modalidade de buscar aumento de visibilidade e possível aumento das métricas de fator de impacto, embora não seja o objetivo principal desse tipo de publicação - uma revisão por pares antecipada e de forma compartilhada.

\section{CONSIDERAÇÕES FINAIS}

Neste texto, ocorre uma breve apresentação do contexto de comunicação científica e dos preprints, meio de publicação em formato pré-publicação. No início do século XXI, houve constantes mudanças nos processos, produtos e serviços voltados para a melhoria da comunicação entre os pesquisadores.

Nesse contexto, existem pesquisadores e servidores de artigos científicos pré-publicação, utilizados, inicialmente, em ciências exatas, em formato ainda não revisado por pares, em acesso aberto e em meio eletrônico. Os preprints estão sendo utilizados também pela área biomédica, adotados, agora, por algumas das principais editoras Cell Press, Nature Publishing Group e pela revista Science, ou seja, existe uma antecipação de leitura de papers futuros. Identifica-se que esse modelo será adotado pelas publicações do SciELO em um novo servidor de preprints.

Nesse contexto, a mudança de paradigma da antecipação da revisão por pares em acesso aberto vem sendo, atualmente, uma das principais discussões em editoração e comunicação científica. Em parte, essa é uma possibilidade utilizada, cujos autores e editores interferem não somente no modelo tradicional de acesso à comunicação científica, mas também nas contribuições ao seu conteúdo a ser publicado e já verificado pela comunidade científica. Segundo Ginsparg (2011), representando uma infraestrutura para a próxima 'geração digital' de pesquisadores, que têm sua experiência de vida já com a cultura de compartilhamento, como já utilizado nas redes sociais e em outras mídias.

Importante destacar que a utilização de DOls por esse modelo é necessária, pois, vincula a publicação e liga-a ao modelo tradicional no pós-publicação, sem a necessidade de atribuição de uma nova ligação. Assim, os DOls são diferentes, mas têm um relacionamento.

Mostra-se que, nos quesitos de disseminação e comunicação 
da informação científica, os servidores de preprints estão sustentados por projetos já utilizados e em constante investimento em visibilidade, como da utilização de métricas relacionadas às mídias sociais e altmetria.

Identificou-se que os principais benefícios dos preprints são: o acesso aberto e imediato a resultados de pesquisa; a divulgação pública de trabalho/projeto/pesquisa recente ou em suas fases de desenvolvimento, pela possibilidade de publicação de versões do preprints; o autor obtém feedback sobre seu trabalho da comunidade científica; controle e interoperabilidade entre os DOls do preprints e da publicação final (artigo); que os autores de biomédicas sentem-se incentivados a publicar em preprints.

Outra informação importante é a de que os preprints são incentivados pelas agências de financiamento internacionais e, consequentemente, nacionais, a curto prazo, pois destinam-se a mostrar os resultados em fases diferentes dos recursos investidos nas pesquisas e também possibilitam informações para o controle dos subsídios.

Volta-se para responder à comunidade científica regional e internacional que o papel da comunicação científica é disponibilizar social e democraticamente a informação produzida pelos pesquisadores mesmo antes da publicação formal.

\section{AGRADECIMENTOS}

Crossref; IBICT; ASAPbio, pelas informações.

INFORMAÇÃO: Uma versão preliminar desse artigo foi publicada em evento promovido pelo IBICT - Encontro de Usuários de Sistemas de Publicação 2017.

Recebido em: 20 dez. 2018. Aceito em: 24 jan. 2019

\section{REFERÊNCIAS}

arXiv. Statistics. 2016.2 Disponível em: <https://arxiv.org/help/stats/2016_by_area/index>. Acesso em: 20 jul. 2018.

ASAPbio. Survey results. 2016. Disponível em: <http://asapbio.org/survey>. Acesso em: 20 mar. 2017.

BILDER, Geoffrey. Members will soon be able to assign Crossref DOls to preprints. Crossref Blog, 5 May 2016. Disponível em: $<$ https://www.crossref.org/blog/members-will-soon-be-able-to-assigncrossref-dois-to-preprints/>. Acesso em: 11 abr. 2017.

bioRXiv. The preprint server for biology. Disponível em: <http://biorxiv.org/>. Acesso em: 20 jul. 2016. 
CALLAWAY, Ewen. Preprints come to life. Nature, 503, 14 Nov. 2013. doi: 10.1038/503180a.

CALLAWAY, Ewen. Heavyweight funders back central site for life-sciences preprints. Nature, v. 542, no. 7641, 13 Fev. 2017. doi: 10.1038/nature.2017.21466.

CROSSREF. Multiple resolution. Disponivel em: <https://www.crossref.org/services/content-registration/multiple-resolution/>. Acesso em: 20 nov. 2018a.

CROSSREF. Preprints. Disponível em: $<$ https://www.crossref.org/services/content-registration/preprints/>. Acesso em: 20 abr. 2018b.

GARVEY, William D.; LIN, Nan; TOMITA, Kazuo. Research studies in scientific communication: IV. The continuity of dissemination of information by "productive scientists". Information Storage and Retrieval, v. 8, n. 6, p. 265-276, 1972. doi: https://doi.org/10.1016/0020-0271(72)90017-4.

GINSPARG, Paul. ArXiv at 20. Nature, v. 476, p. 145-147, 11 Aug. 2011. doi: $10.1038 / 476145 a$.

KLAUS, Bernd; DEL ALAMO, David. Talent Identification at the limits of Peer Review: an analysis of the EMBO Postdoctoral Fellowships Selection Process. bioRxiv, p. 481655, 2018.

MEADOWS, A. J. A comunicação científica. Tradução de Antonio Agenor Briquet de Lemos. Brasília, DF: Briquet de Lemos, 1999.

MUELLER, Suzana Pinheiro Machado. A Comunicação Científica e o movimento de acesso livre ao conhecimento. Ciência da Informação, Brasília, DF, v. 35, n. 2, p. 27-38, 2006.doi: https://doi.org/10.1590/s010019652006000200004.

PACKER, Abel L.; SANTOS, Solange; MENEGHINI, Rogerio. SciELOPreprints a caminho. SciELO em Perspectiva, 22 fev. 2017. Disponível em: <http://blog.scielo.org/blog/2017/02/22/scielo-preprints-acaminho>. Acesso em: 11 abr. 2017.

POWELL, Kendall. Does it take too long to publish research? Nature, 530, p.148-151, 11 Fev. 2016. doi: 10.1038/530148a.

PREPRINTS.ORG. Disponível em: <https://www.preprints.org/about>. Acessoem: 20 jun. 2018.

PUBLONS. Track more of your research impact. Disponível em: $<$ https://publons.com/about/home/>. Acesso em: 20 out. 2018.

STAT. Stat News.Should researchers publish their findings before peer review? Disponível em: <https://www.statnews.com/2016/05/27/preprintbiology-bioxriv/>. Acesso em: 27 maio 2016.

WERSIG, G. Information Science: the study of post modern knowledge usage. Information Processing \& Management, Amsterdam, v. 29, n. 2, 
p. 229-239, 1993. doi: https://doi.org/10.1016/0306-4573(93)90006-y.

WHITIFIELD, John. Open Access comes of age. Nature, v. 474, n. 428, 2011. doi: $10.1038 / 474428 a$. 